\title{
Exploring Time Domain Multi-Messenger Astronomy through the Virtual Observatory
}

\author{
Ada Nebot ${ }^{1}$, Mark G. Allen, Pierre Fernique, Matthieu Baumann, Thomas Boch, \\ Caroline Bot, Sebastien Derriere, Françoise Genova, Katharina Lutz \\ CDS, Université de Strasbourg, CNRS, Observatoire astronomique de Strasbourg, UMR 7550 \\ 11 rue de l'Université, 67000 Strasbourg, France \\ E-mail: ada.neboteastro.unistra.fr \\ E-mail: mark.allen@astro.unistra.fr \\ E-mail: pierre.ferniquedastro.unistra.fr \\ E-mail: matthieu.baumanneastro.unistra.fr \\ E-mail: thomas.bocheastro.unistra.fr \\ E-mail: caroline.boteastro.unistra.fr \\ E-mail: sebastien.derrieredastro.unistra.fr \\ E-mail: francoise.genovadastro.unistra.fr \\ E-mail: katharina.lutz@astro.unistra.fr
}

\section{Dave Morris}

Institute for Astronomy, School of Physics and Astronomy

University of Edinburgh, Royal Observatory, Blackford Hill, Edinburgh, EH9 3HJ, UK

E-mail: dmceroe.ac.uk

What do Cepheid stars, Binary Neutron stars, Supernovae explosions and Active Galactic Nuclei have in common? They are all variable objects, although the physical processes and the associated time-scales that govern their variability can be very different (pulsations, collapse, accretion, bursts, ... with characteristic time-scales from milliseconds to hundreds of years). Some events can trigger follow-up observations and a fast reaction to those events can be crucial to determining the nature of the object. To better understand the nature of these objects and the mechanism behind the source of variation a multi-wavelength and even multi-messenger approach is often needed. In 2017 for the first time there was a direct detection of gravitational waves and electromagnetic emission from colliding neutron stars. The discovery involved more than 70 ground and space based telescopes and more than 3000 astronomers. GW170817 beyond being of great relevance by its own illustrates at the highest level the need from the Time Domain Astronomy community to transmit events, collect and share information and to coordinate observations. The Virtual Observatory (VO) should match the common needs of the different scientific use cases. In this paper, we give some highlights of the work on enabling Time Domain Astronomy in the VO, and coordinating the international effort in IVOA, part of which has been done within the ASTERICS DADI package. This includes standards for spatial and temporal coverage,

\section{$1 \quad$ Speaker}

(c) Copyright owned by the author(s) under the terms of the Creative Commons 
and Time Domain data as will be needed for Time Domain Multi-Messenger Astronomy. Other highlights include prototype visualisation tools taking into account the temporal dimension, and tools for fast cross-matching.

The New Era of Multi-Messenger Astrophysics - Asterics2019 


\section{Introduction}

Time Domain Astronomy is currently a scientific priority of the International Virtual Observatory Alliance (IVOA). The IVOA is the international initiative that coordinates the development of astronomical interoperability standards.

The most common data challenges related to Time Domain Multi-Messenger Astronomy are the following:

- Multi-wavelength/messenger approach

- Transmission of alerts and follow-up observations

- Visualisation \& navigation thought the data

- Coordination \& transmission of information

The Virtual Observatory, and tools provided to astronomers, should match the common needs of the different scientific use cases. In the following sections, we will give some highlights of the ASTERICS DADI work on enabling Time Domain Astronomy in the VO, and on how we are coordinating the international effort in IVOA, and information about other relevant developments, in particular at the Strasbourg astronomical Data Centre CDS.

\section{Multi-wavelength/messenger approach}

To combine data from different missions covering different wavelengths fast and efficient cross-matching techniques are developed (see e.g. http://cdsxmatch.u-strasbg.fr). Crossmatching tools take into account mostly only positional information. While cross-matching tools have been developed to take into account the multi-wavelength aspect of sources [1], the temporal dimension has not yet been fully integrated. For fast moving objects the location in the sky at two different moments varies and a simple positional cross-match could lead to erroneous results. Information on the time of observation and on the proper motion is needed in order to perform a proper cross-match. A possible enhancement for cross-matching capabilities would be to add the temporal dimension as extra information.

Source characterization and classification on the basis of their variability in a multiwavelength space and dealing with large and heterogeneous data using machine learning or deep learning techniques are questions that are being discussed within the IVOA Knowledge Discovery Interest Group (https://wiki.ivoa.net/twiki/bin/view/IVOA/IvoaKDD).

\section{Alerts and follow-up observations}

Transient events can trigger alerts for which follow-up observations are crucial for identification of the source. While some transient events fade out quickly in a specific wavelength, they might still be visible at other wavelengths. Generation and transmission of such alerts between telescopes in an automated way can be done through the IVOA standard VOEvent [2] and its Transmission Protocol [3]. These two protocols are nowadays widely used by the community, from Fast Radio Bursts [4] to Solar and Planetary Sciences [5]. Public LIGO/Virgo alerts are also distributed as VOEvents ${ }^{2}$.

2 https://emfollow.docs.ligo.org/userguide/content.html 


\section{Visualisation \& navigation through the data}

Hierarchical Progressive Survey (HiPS) [6] and Multi-Order Coverage map (MOC) [7], both IVOA specifications widely used by the community, allow the users to quickly navigate through datasets in space (collection of images or catalogues). HiPS is a hierarchical scheme for the description, storage and access of sky survey data, which allows the possibility of creating HiPS-cubes where the third dimension typically used is the wavelength, however nothing prevents users to create HiPS-cubes where the third dimension is a temporal one to create a time-view of the sky. Based on a hierarchical approach of the sky a MOC gives the approximate area covered by different missions, enabling the capacity of comparing coverage maps of large datasets in a seamless way. Users can make fast operations such as intersections and unions of several missions.

Based on the same technology as MOC, time-MOCs, T-MOCs take into account the time dimension of data, allowing users to see if two data sets overlapped in time in a very fast way. In Figure 1 we use Aladin to visualize a certain region of the sky covered by a particular catalogue. In the upper panel we show the source positions in that catalogue in red as well as the spatial coverage (MOC) in green. In the bottom panel we show the $\mathrm{V}$ magnitude over the date of observation for the same sources in red and the temporal coverage of the catalogue (T-MOC) in green. Ideally space and time will be combined to create a unique coverage map, a space-time MOC (ST-MOC) $)^{3}$.

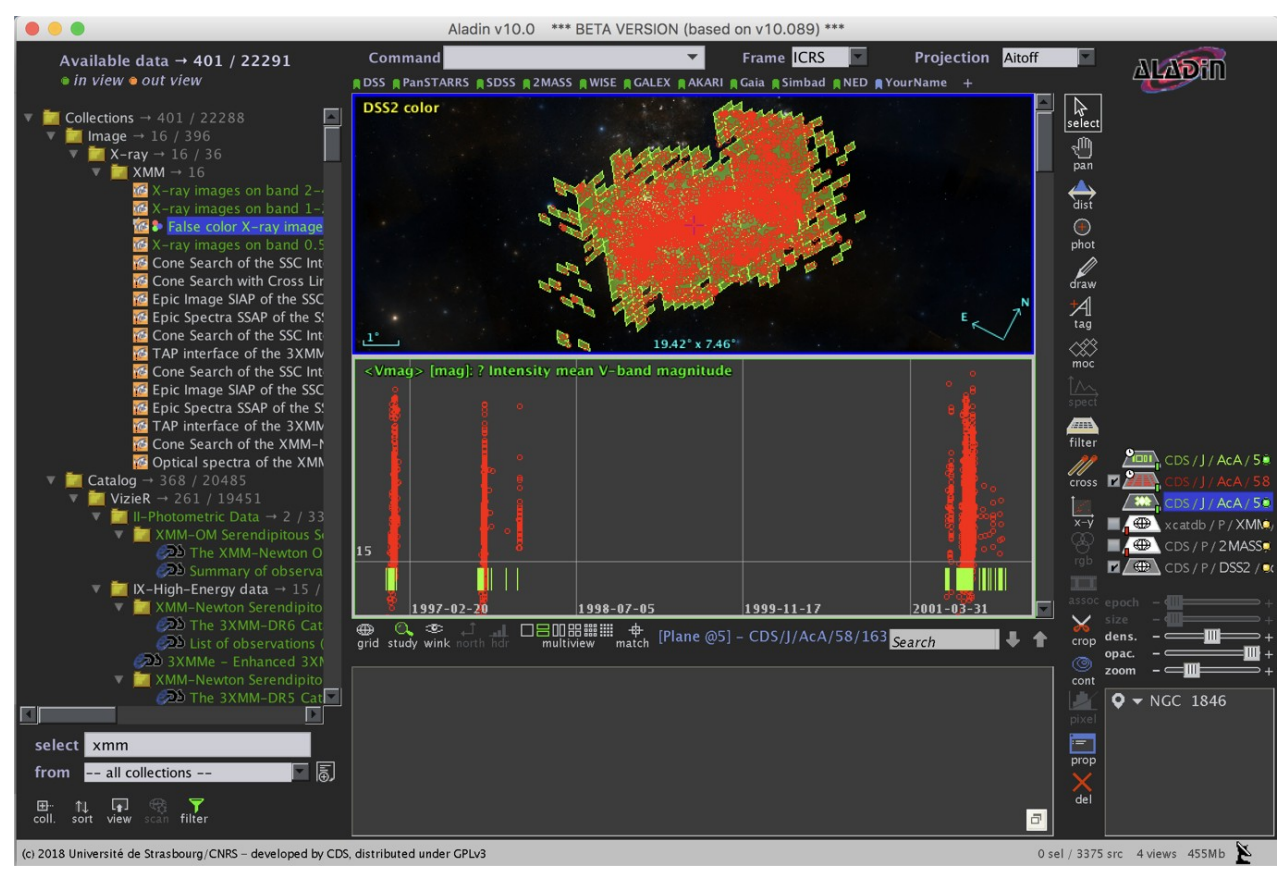

Figure 1. Visualisation of a catalogue in Aladin with the space (top) and the time (bottom) coverages shown in green.

\section{Visualization of light-curves}

Since then we have developed a way to connect both space and time coverages: ST-MOC. An IVOA Note can be found under: http://ivoa.net/documents/stmoc/index.html. Information on usage and demo videos are currently available through $\underline{\mathrm{http}}$ ://aladin.u-strasbg.fr/java/stmoc/index.html 
A quick look at the spectral energy distribution of a source can bring light to its nature. With this idea in mind, photometric viewers are widely used. Based on the location on the sky and a search radius around that position, fluxes are displayed against frequency, for data in well defined filters ${ }^{4}$. Information on filter characterization has been collected in a standardized way ${ }^{5}$.

A quick look at the light-curve of an object can also help to learn about its nature. Ideally photometric viewers would have the capability to include the time as a possible extra dimension, to become a light-curve viewer. In a first step a user would perform a cone search around a position and within a certain interval of time. The key point for being able to create such a tool or service is to have a standard annotation for time ${ }^{6}$ and to transform the time values into a pivot format in which the data can be compared. Within the VO framework we are working towards such an implementation. Due to the sparse nature of the data when plotting data taken years apart, we are investigating different ways of accessing and representing data in a hierarchical way.

\section{Coordination \& transmission of information}

Planning observations involves knowledge on target visibility, telescope time availability, and prioritization of the scheduled time. Different visibility and schedule tools exist but they have inputs and outputs which are very heterogeneous. Some level of standardization would facilitate the planning and organization of observations. With that aim two protocols are being developed within the IVOA framework: an Object Visibility Simple Access Protocol ${ }^{7}$ and an Observation Locator Table Access Protocol ${ }^{8}$. A facility database is also being built with the aim of documenting observatories, locations, telescopes, instruments, filters, etc. that are (or have been) available to the community [8]. In this context, one of the ideas that is also being discussed and prototyped is the possibility of having an exchange platform dedicated to such type of follow-up.

\section{Conclusion}

Time Domain Astronomy is a scientific priority of the IVOA. Data challenges related to Time Domain Multi-Messenger Astronomy involve multi-wavelength/messenger approach, fast transmission of alerts and follow-up observations, providing tools for rapid and easy visualisation and navigation thought the data and finally soothing coordination \& transmission of information in international collaborations. IVOA standards have been and continue to be developed to facilitate the astronomers' and facility managers' experience in all these aspects. In this paper we have summarised some of the standards relevant for Time Domain Astronomy, a comprehensive list of all the IVOA standards can be accessed through the IVOA Documents repository under http://ivoa.net/documents/.

\footnotetext{
$4 \quad$ See e.g. http://vizier.u-strasbg.fr/vizier/sed/ See the filter profile system of the SVO http://svo2.cab.inta-csic.es/svo/theory/fps/ http://ivoa.net/documents/Notes/TimeSys/index.html http://ivoa.net/documents/ObjVisSAP/index.html http://ivoa.net/documents/ObsLocTAP/index.html
} 


\section{References}

[1] Motch, C. et al., The ARCHES Project, 2017ASPC..512..165M

[2] Seaman, R. et al, Sky Event Reporting Metadata Version 2.0, DOI: 10.5479/ADS/bib/2011ivoa.spec.0711S

[3] Swinbank, J. D.; Allan, A.; Denny, R. B., VOEvent Transport Protocol Version 2.0, DOI: 10.5479/ADS/bib/2017ivoa.spec.0320S

[4] Petroff et al., VOEvent Standard for Fast Radio Bursts, 2017arXiv171008155P

[5] Cecconi et al., VOEvent for Solar and Planetary Sciences, 2018arXiv181112680C

[6] Fernique et al., HiPS - Hierarchical Progressive Survey Version 1.0, DOI: 10.5479/ADS/bib/2017ivoa.spec.0519F

[7] Fernique et al., MOC - HEALPix Multi-Order Coverage map Version 1.0, DOI : 10.5479/ADS/ bib/2014ivoa.spec.0602F

[8] Perret, et al. Shared nomenclature and identifiers for telescopes and instruments, DOI: 10.1051/ epjconf $/ 201818604002$

\section{Aknowledgements}

The authors acknowledge support from ASTERICS, a project funded by the European Commission under the Horizon2020 programme (id 653477), in the framework of ASTERICS Work Package 4 Data Access, Discovery and Interoperability. 